chamber system, one compartment was closed and connected to a constant-pressure device. A fairly permeable cellulose filter-membrane (effective area for heavy water diffusion about 30 per cent) separated the two compartments. A constant known flow of water could be obtained and the permeation of a test substance against the flow could be measured. The accompanying graph shows the result for deuterium-labelled water as the test substance. The total flux of water against the mass flow is plotted against the net flux, which, in this case, is equal to the velocity of the streaming water times the area available for flow. The curve is plotted from the relation:

$$
\Phi_{\text {out }}=\frac{\Delta w}{\exp \left(\Delta w \cdot \delta / A R T u^{\prime}\right)-1},
$$

where $\Phi_{\text {out }}$ is the total flux of water out, $\Delta w$ is the net flux of water, $A$ is the area available for flow, $R$ the gas constant, $T$ the absolute temperature and $u^{\prime}$ the mobility of the water molecule in the membrane, in this case taken to be equal to $D_{w} / R T$, where $D_{w}$ is the free diffusion coefficient of water in water. $\delta$ is equal to the membrane thickness. The experimental observations seem to follow the theoretical curve quite satisfactorily (see figure).

A full report of these experiments and others in progress will be published later.

Institute of Physiology,

University of Uppsala. Nov. 6.

'Manegold, E., and Solf, K., Kolloid-Z., 59, 179 (1932). Williams, J. W.' and Cady, L. C., Chem. Rev., 14, 131 (1934). Bloch, I, Bull'. Math. Biophys., 8, 21 (1946). Rashevsky , N., "Mathematical
Biophysics" (rev, edit., Chicago, 1948). Ussing, H. H., Acta Bhysiol. Scand., 19, 43 (1949). Teorell, T., Z. Electrochem., 55, 460 (1951).

${ }^{2}$ Hertz, G., Phys. Z., 23, 433 (1922) ; Z. Phys., 19, 35 (1923).

' Hertz, G., Phys. $Z$., 23,433
${ }^{3}$ Teorell, T. (to be published).

"Ussing, H. H., in F. F. Nord, "Advances in Enzymology", 13 (1952).

"Teorell, T., in "Progress in Biophysics", 3 (London, 1953).

\section{Sidereal Correlation of High-Energy Cosmic Rays}

Extensive air showers have been recorded at Auckland (altitude $40 \mathrm{~m}$.) during the period February 1951-February 1952 inclusive, with three counter trays each of sensitive area $2,360 \mathrm{~cm}^{2}$ placed at the corners of an equilateral triangle with $4 \mathrm{~m}$. sides. About $3 \times 10^{5}$ events have been recorded. Averaged over the year, the results show a solar diurnal variation of $1.46 \pm 0 \cdot 16$ per cent (first Fourier amplitude with statistical probable error), with the maximum at $2.9 \pm 0.4 \mathrm{hr}$. local solar time. This correlates well with temperature.

When the number of showers occurring in halfhourly intervals of sidereal time is averaged over the year, the figures show a sidereal variation with first Fourier amplitude $1.06 \pm 0 \cdot 16$ per cent with the maximum at $19 \cdot 3 \pm 0 \cdot 6 \mathrm{hr}$. local sidereal time. This result is similar to the Fourier amplitude of $0.39 \pm$ $0 \cdot 13$ per cent with a maximum at $22 \pm 1 \cdot 3 \mathrm{hr}$. local sidereal time obtained by Daudin and Daudin ${ }^{1}$ in 1951 at the Pic du Midi for extensive air showers recorded with a different arrangement of counters.

A seasonal variation in the solar diurnal effect would give an apparent sidereal effect when the results are expressed in sidereal time and averaged over the year. In this case, provided the solar seasonal effect is the same in the southern as in the northern hemisphere, we would expect the apparent sidereal variation obtained in the two hemispheres to differ in phase by $180^{\circ}$. In fact, the variations observed at Auckland (lat. $37^{\circ} \mathrm{S}$.) and the Pic du Midi (lat. $43^{\circ}$ N.) are in phase, and the maxima coincide in local sidereal time almost within the probable error. This gives grounds for believing that the variation observed is a genuine sidereal effect and is not a residuum due to seasonal changes in the solar diurnal variation.

Although the times of the maxima at Auckland and the Pic du Midi do not agree exactly, at both places the local maximum coincides closely with the time at which the Milky Way is overhead. This suggests that cosmic rays come preferentially from regions of high star density.

At Auckland the maximum is not far from the time ( $18 \mathrm{hr}$. local sidereal time) at which the centre of the Galaxy crosses the meridian. The declination of the galactic centre is $-26^{\circ}$. This means that it passes daily within $11^{\circ}$ of the vertical at Auckland, but only within $69^{\circ}$ of the vertical at the Pic du Midi. Any effect due to cosmic rays coming from the central region of the Galaxy should therefore be much stronger in Auckland than in Europe. This accords with the fact that our sidereal amplitude is nearly three times as large as that obtained at the Pic du Midi. The results therefore indicate that more highenergy cosmic rays are observed when the centre of the Galaxy is overhead.

We cannot reconcile these results with those of Ballario, Brunelli, de Marco and Martelli2, who observed dense showers on the Testa Grigia for a period of three months and obtained a maximum at about $10 \mathrm{hr}$. Central European Time, which we find corresponds to $5 \mathrm{hr}$. local sidereal time.

One of us (J. R. S.) is indebted for a fellowship to the University of New Zealand Research Committee, which also gave a grant covering the major cost of the apparatus.

Department of Physics, F. J. M. FARLEY J. R. STOREY

Auckland University College,

Auckland, C.1, New Zealand.

$$
\text { Dec. } 11 .
$$

1 Daudin, A., and Daudin, M. J., C.R. Acad. Sci., Paris, 284, 1551 (1952).

2 Ballario, C., Brunelli, B., de Marco, A., and Martelli, G., Nuovo Cim., 8, 587 (1951); see also Phys. Rev., 83, 666 (1951).

\section{Motion of the Storm-D Regions}

W. R. PiggotrT ${ }^{1}$ has reported coherent variations with longitude which he interprets as motion of the "storm- $D$ regions" once around the earth in $48 \mathrm{hr}$. This result is based on the data of about three years from each of six vertical-incidence ionospheric stations. Work at the National Bureau of Standards, however, based on ionospheric data tabulations from seventeen stations for the four years, 1949-52, does not support the hypothesis of coherent motion of such regions.

Reference will be made here to (per cent) 'probability of blackout' rather than to storm- $D$ regions. Diurnal variation of blackout probability is determined by counting the number of instances (at each hour for the four-year period) of excessive absorption. In these cases the letter $B$ is the standard symbol used to replace a numerical value for all the ionospheric parameters normally scaled: $f 0 F^{2}, h^{\prime} F 2$, fOF $1, h^{\prime} F 1, f 0 E, h^{\prime} E$.

Blackout may often be a misnomer, since the noecho conditions can also arise if the equipment lacks 